Article

\title{
Adaptive-Gain Second-Order Sliding Mode Direct Power Control for Wind-Turbine-Driven DFIG under Balanced and Unbalanced Grid Voltage
}

\author{
Yaozhen Han * and Ronglin Ma * \\ School of Information Science and Electrical Engineering, Shandong Jiaotong University, Jinan 250357, China \\ * Correspondence: hyz125@163.com (Y.H.); maronglin@sdjtu.edu.cn (R.M.)
}

Received: 26 August 2019; Accepted: 10 October 2019; Published: 14 October 2019

\begin{abstract}
In a wind turbine system, a doubly-fed induction generator (DFIG), with nonlinear and high-dimensional dynamics, is generally subjected to unbalanced grid voltage and unknown uncertainty. This paper proposes a novel adaptive-gain second-order sliding mode direct power control (AGSOSM-DPC) strategy for a wind-turbine-driven DFIG, valid for both balanced and unbalanced grid voltage. The AGSOSM-DPC control scheme is presented in detail to restrain rotor voltage chattering and deal with the scenario of unknown uncertainty upper bound. Stator current harmonics and electromagnetic torque ripples can be simultaneously restrained without phase-locked loop (PLL) and phase sequence decomposition using new active power expression. Adaptive control gains are deduced based on the Lyapunov stability method. Comparative simulations under three DPC schemes are executed on a 2-MW DFIG under both balanced and unbalanced grid voltage. The proposed strategy achieved active and reactive power regulation under a two-phase stationary reference frame for both balanced and unbalanced grid voltage. An uncertainty upper bound is not needed in advance, and the sliding mode control chattering is greatly restrained. The simulation results verify the effectiveness, robustness, and superiority of the AGSOSM-DPC strategy.
\end{abstract}

Keywords: DFIG; adaptive-gain second-order sliding mode; direct power control; balanced and unbalanced grid voltage

\section{Introduction}

Over the past decade, renewable energy generation has continued to grow rapidly due to widely known problems such as environmental pollution and resource shortage [1]. Wind power generation accounted for $21 \%$ of renewable generating capacity until the end of 2018 [2]. The doubly-fed induction generator (DFIG) has become the most widely used electric generator in wind turbine systems, owing to its inherent advantages including high system efficiency, low converter rating, four-quadrant active and reactive power capability, variable wind speed operation, and controllable power factor [3,4].

DFIG control is one of the most difficult issues in a wind turbine system because DFIG dynamics is intrinsically nonlinear and high-dimensional; system model parameters are uncertain; and the encountered wind speed is random [5,6]. Currently, the main control techniques for DFIG in industrial application are vector control (VC) and direct power control (DPC) [7]. Although the VC method has outstanding steady-state performance, its dynamic performance is rather disillusionary due to the hysteresis of proportional integral (PI) control [8]. Many studies improved the traditional VC [9-12], but some inherent problems still exist, such as that the control algorithm is complex, and synchronous coordinate transform and phase-locked loop (PLL) are still prerequisites [13,14].

DPC is a useful alternative to improve dynamic performance. Current rotor control loops are not required for a DPC scheme, and a switching table is directly used to select a suitable switching 
vector. Hence, the control structure is easy to implement, and excellent dynamic performance is achieved [15]. Many modified achievements have been published based on this traditional DPC idea [16-18]. However, the switching frequency is unfixed, unacceptable power ripples still exist, and system robustness should be improved [19].

Among all the nonlinear control approaches, sliding mode control, which is used in wind turbine systems, is a robust control method capable of providing finite-time convergence, disturbance suppression, fast response, and simple implementation $[20,21]$. Some studies have evaluated the direct power sliding mode control for DFIG [22-27]. Although good results were achieved, some drawbacks still exist, and positive and negative sequence decomposition is needed [23]; all these studies focused on the conventional first-order sliding mode (FOSM), with unsatisfactory control switching and variable switching frequency. These intrinsic drawbacks caused by the FOSM may produce torque ripple, harmonic current, overheating of the windings, etc. [28].

The well-known super-twisting second order sliding mode (SOSM)method hides a discontinuous item under the integral and generates continuous control action to propel the sliding mode vector and its derivative to the origin in finite time, and then the control chattering can be greatly attenuated [29]. Some literature discussed the applications for DFIG [30-32], which need synchronous rotating frame transformation and were mainly concentrated on SOSMVC control.

In recent years, some scholars set about studying the second-order sliding mode(SOSM) DPC under stationary reference frames. Susperregui et al. [33] proposed a fixed-frequency PWM-based rotor converter control and a reactive power control under balanced grid voltage. In the study following [33], Reference [34] achieved power regulation and grid synchronization based on a SOSM control scheme. Yet, it was also verified under a balanced grid voltage. Martinez presented a SOSM global control scheme for DFIG suffering from unbalanced and distorted grid voltage [35]. Both rotor side and grid side power converters were controlled via a super-twisting algorithm. However, the mechanism of dealing with unbalanced grid voltage condition was not mentioned and the upper bound of uncertainty, which cannot be easily estimated in many practical cases, was hypothetically known. Reference [36] presented a super-twisting DPC scheme for adjusting active and reactive power in detail. Yet, the strategy only focused on a balanced voltage scenario. Unbalanced grid voltage, natural flux, and uncertainty upper bound conditions were not considered. As the continuity of the study presented in Reference [36], the controller in Reference [37] was designed with adaptive control gain to handle the unknown uncertainty upper bound. Yet, the unbalanced grid voltage condition, which may cause a severe power harmonic, was still undiscussed.

As mentioned above, some key issues should be considered simultaneously in a DPC scheme, including the following: (a) The scheme should deal with the uncertainty upper bound scenario. The overestimation of uncertainty can produce redundant control gain. (b) Switching frequency is fixed and control chattering can be significantly restrained. (c) The scheme needs a simple control structure, needless of phase sequence decomposition, coordinate transformation, and PLL. (d) The scheme should be valid for both balanced and unbalanced grid voltage, and stator current harmonic and electromagnetic torque ripple can be restrained simultaneously. (e) The scheme needs to filter the roughly static stator flux component. Hence, this paper proposes an adaptive-gain SOSM (AGSOSM-DPC) scheme for DFIG subjected to balanced and unbalanced grid voltage. First, a new active power expression is applied based on a detailed phase sequence analysis. Then, an adaptive control gain law of SOSM DPC is designed via the Lyapunov stability method to solve the unknown upper bound of uncertainty. A band-pass filter was also used to calculate stator flux and avoid stator natural flux. The main contributions of the paper include the following: (1) Under a two-phase stationary reference frame, a novel AGSOSM-DPC strategy for DFIG is proposed that can solve the abovementioned key issues simultaneously; (2) using adaptive control gain, the upper bound of uncertainty is not necessarily known in advance; and (3) rotor voltage control chattering is highly suppressed via a super-twisting algorithm. 
The rest of the paper is organized as follows: An elaborated model analysis is presented as Section 2 . Section 3 presents the controller design and stability analysis. Section 4 shows the comparative simulation results obtained on a 2-MW DFIG. Finally, Section 5 provides the conclusions.

\section{Model Analysis}

The diagrammatic drawings of DFIG-based wind turbine system and DFIG equivalent circuit under a two-phase stationary reference frame are shown in Figure 1. To facilitate the analysis and design, the DFIG equivalent model under two-phase stationary frame can be presented as follows [22]:

$$
\left\{\begin{array}{l}
\boldsymbol{\psi}_{s \alpha \beta}=L_{s} \boldsymbol{I}_{s \alpha \beta}+L_{m} \boldsymbol{I}_{r \alpha \beta} \\
\boldsymbol{\psi}_{r \alpha \beta}=L_{r} \boldsymbol{I}_{r \alpha \beta}+L_{m} \boldsymbol{I}_{s \alpha \beta} \\
\boldsymbol{U}_{s \alpha \beta}=R_{s} \boldsymbol{I}_{s \alpha \beta}+\frac{d \boldsymbol{\psi}_{s \alpha \beta}}{d t} \\
\boldsymbol{V}_{r \alpha \beta}=R_{r} \boldsymbol{I}_{r \alpha \beta}+\frac{d \boldsymbol{\psi}_{r \alpha \beta}}{d t}-j \omega_{r} \boldsymbol{\psi}_{r \alpha \beta} \\
\boldsymbol{T}_{e}=\frac{3}{2} p \operatorname{Im}\left\{\boldsymbol{\psi}_{s \alpha \beta}^{*} \boldsymbol{I}_{s \alpha \beta}\right\} \\
\boldsymbol{S}_{s}=\boldsymbol{P}_{s}+j \boldsymbol{Q}_{s}=\frac{3}{2} \boldsymbol{I}_{s \alpha \beta}^{*} \boldsymbol{U}_{s \alpha \beta}
\end{array}\right.
$$

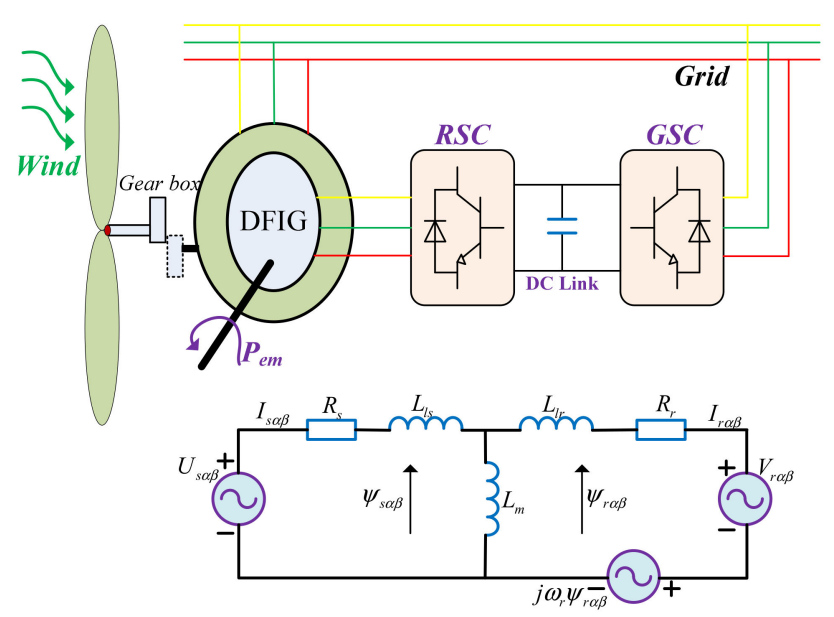

Figure 1. Doubly-fed induction generator (DFIG)-based wind power system schematic diagram and DFIG equivalent circuit under a two-phase stationary coordinate frame.

Although phase sequence decomposition is not needed in the proposed control scheme, both positive and negative sequence components of stator voltage and current are listed to analyze the effect of DPC when DFIG is subjected to unbalanced grid voltage.

$$
\begin{gathered}
\left\{\begin{array}{l}
\boldsymbol{U}_{s \alpha \beta}=u_{s \alpha}+\mathrm{j} u_{s \beta}=\boldsymbol{U}_{s \alpha \beta}^{+}+\boldsymbol{U}_{s \alpha \beta}^{-} \\
\boldsymbol{U}_{s \alpha \beta}^{+}=u_{s \alpha}^{+}+\mathrm{j} u_{s \beta^{\prime}}^{+}, \boldsymbol{U}_{s \alpha \beta}^{-}=u_{s \alpha}^{-}+\mathrm{j} u_{s \beta}^{-} \\
u_{s \alpha}^{+}=\left|\boldsymbol{U}_{s \alpha \beta}^{+}\right| \cos \left(\omega_{s} t+\theta_{u}^{+}\right), u_{s \beta}^{+}=\left|\boldsymbol{U}_{s \alpha \beta}^{+}\right| \sin \left(\omega_{s} t+\theta_{u}^{+}\right) \\
u_{s \alpha}^{-}=\left|\boldsymbol{U}_{s \alpha \beta}^{-}\right| \cos \left(\omega_{s} t+\theta_{u}^{-}\right), u_{s \beta}^{-}=\left|\boldsymbol{U}_{s \alpha \beta}^{-}\right| \sin \left(\omega_{s} t+\theta_{u}^{-}\right)
\end{array}\right. \\
\left\{\begin{array}{l}
\boldsymbol{I}_{s \alpha \beta}=i_{s \alpha}+\mathrm{j} i_{s \alpha}=\boldsymbol{I}_{s \alpha \beta}^{+}+\boldsymbol{I}_{s \alpha \beta}^{-} \\
\boldsymbol{I}_{s \alpha \beta}^{+}=i_{s \alpha}^{+}+\mathrm{ji}_{s \beta}^{+} \boldsymbol{I}_{s \alpha \beta}^{-}=i_{s \alpha}^{-}+\mathrm{ji} i_{s \beta}^{-} \\
i_{s \alpha}^{+}=\left|\boldsymbol{I}_{s \alpha \beta}^{+}\right| \cos \left(\omega_{s} t+\theta_{i}^{+}\right), i_{s \beta}^{+}=\left|\boldsymbol{I}_{s \alpha \beta}^{+}\right| \sin \left(\omega_{s} t+\theta_{i}^{+}\right) \\
i_{s \alpha}^{-}=\left|\boldsymbol{I}_{s \alpha \beta}^{-}\right| \cos \left(\omega_{s} t+\theta_{i}^{-}\right), i_{s \beta}^{-}=\left|\boldsymbol{I}_{s \alpha \beta}^{-}\right| \sin \left(\omega_{s} t+\theta_{i}^{-}\right)
\end{array}\right.
\end{gathered}
$$


where $\left|\boldsymbol{U}_{s \alpha \beta}^{+}\right|,\left|\boldsymbol{U}_{s \alpha \beta}^{-}\right|,\left|\boldsymbol{I}_{s \alpha \beta}^{+}\right|$, and $\left|\boldsymbol{I}_{s \alpha \beta}^{-}\right|$are the amplitudes of positive and negative sequence components of stator voltage and stator current. The values $\theta_{u}^{+}, \theta_{u}^{-}, \theta_{i}^{+}$, and $\theta_{i}^{-}$are the corresponding initial phase angles.

Substituting Formulas (2) and (3) into Formula (1), the stator instantaneous power can be deduced as follows:

$$
\left\{\begin{array}{l}
P_{s}=P_{s 0}+P_{s 1}+P_{s 2} \\
Q_{s}=Q_{s 0}+Q_{s 1}+Q_{s 2}
\end{array},\right.
$$

where $P_{s 0}$ and $Q_{s 0}$ are the average values of active and reactive power; $\boldsymbol{P}_{s 1}, \boldsymbol{P}_{s 2}, Q_{s 1}$, and $Q_{s 2}$ are the oscillating components at twice the grid frequency of active and reactive power, respectively. According to Formulas (2) and (3), these oscillating components can be represented as follows:

$$
\begin{gathered}
\left\{\begin{array}{l}
\boldsymbol{P}_{s 1}=\frac{3}{2}\left(u_{s \alpha}^{+} i_{s \alpha}^{-}+u_{s \beta}^{+} i_{s \beta}^{-}\right)=\frac{3}{2}\left|\boldsymbol{U}_{s \alpha \beta}^{+} \| \boldsymbol{I}_{s \alpha \beta}^{-}\right| \cos \left(2 \omega_{s} t+\theta_{u+}-\theta_{i-}\right) \\
\boldsymbol{P}_{s 2}=\frac{3}{2}\left(u_{s \alpha}^{-} i_{s \alpha}^{+}+u_{s \beta}^{-} i_{s \beta}^{+}\right)=\frac{3}{2}\left|\boldsymbol{U}_{s \alpha \beta}^{-} \| \boldsymbol{I}_{s \alpha \beta}^{+}\right| \cos \left(2 \omega_{s} t+\theta_{i+}-\theta_{u-}\right)
\end{array}\right. \\
\left\{\begin{array}{l}
\boldsymbol{Q}_{s 1}=\frac{3}{2}\left(u_{s \beta}^{+} i_{s \alpha}^{-}-u_{s \alpha}^{+} i_{s \beta}^{-}\right)=\frac{3}{2}\left|\boldsymbol{U}_{s \alpha \beta}^{+} \| \boldsymbol{I}_{s \alpha \beta}^{-}\right| \sin \left(2 \omega_{s} t+\theta_{u+}-\theta_{i-}\right) \\
\boldsymbol{Q}_{s 2}=\frac{3}{2}\left(u_{s \beta}^{-} i_{s \alpha}^{+}-u_{s \alpha}^{-} i_{s \beta}^{+}\right)=\frac{3}{2}\left|\boldsymbol{U}_{s \alpha \beta}^{-} \| \boldsymbol{I}_{s \alpha \beta}^{+}\right| \sin \left(2 \omega_{s} t+\theta_{i+}-\theta_{u-}\right)
\end{array}\right.
\end{gathered}
$$

This clearly indicates that $\boldsymbol{P}_{s 1}, Q_{s 1}$ and $\boldsymbol{P}_{s 2}, Q_{s 2}$, caused by positive sequence voltage and negative sequence current and negative sequence voltage and positive sequence current, respectively, are the oscillating parts with twice the grid frequency. A third harmonic current is generated and causes severe harmonic distortion if active and reactive power are both simultaneously maintained as a constant under the unbalanced grid voltage condition.

Considering Formulas (2) and (3), the electromagnetic torque can be expressed as follows:

$$
T_{e}=T_{e 0}+T_{e 1}+T_{e 2}
$$

where $T_{e 0}$ is the average value of electromagnetic torque; $T_{e 1}$ and $T_{e 2}$ are the oscillating parts with twice the grid frequency. To neglect the effect of stator resistance, $T_{e 1}$ and $T_{e 2}$ can be denoted as follows:

$$
\left\{\begin{array}{l}
\boldsymbol{T}_{\boldsymbol{e} 1}=\frac{3}{2 \omega_{1}}\left(u_{s \alpha}^{+} i_{s \alpha}^{-}+u_{s \beta}^{+} i_{s \beta}^{-}\right)=\frac{3}{2 \omega_{1}}\left|\boldsymbol{U}_{s \alpha \beta}^{+}\right|\left|\boldsymbol{I}_{s \alpha \beta}^{-}\right| \cos \left(2 \omega_{s} t+\theta_{u+}-\theta_{i-}\right) \\
\boldsymbol{T}_{\boldsymbol{e} 2}=\frac{3}{2 \omega_{1}}\left(u_{s \alpha}^{-} i_{s \alpha}^{+}+u_{s \beta}^{-} i_{s \beta}^{+}\right)=\frac{3}{2 \omega_{1}}\left|\boldsymbol{U}_{s \alpha \beta}^{-}\right|\left|\boldsymbol{I}_{s \alpha \beta}^{+}\right| \cos \left(2 \omega_{s} t+\theta_{i+}-\theta_{u-}\right)
\end{array}\right.
$$

In Formula (8), it is clearly indicated that $T_{e 1}$ and $T_{e 2}$ are caused by positive sequence voltage and negative sequence current, and negative sequence voltage and positive sequence current, respectively, and are the oscillating parts with twice the grid frequency, which may generate bearing chattering and influence the service life.

To achieve active and reactive power tracking under balanced grid voltage, and also to suppress stator current harmonics and electromagnetic torque ripples under unbalanced grid voltage, a new active power expression is used to track instead of the traditional active expression [18,25,38]. The new active power can be expressed as follows:

$$
\boldsymbol{P}_{s n}=-\frac{3}{2} \operatorname{Im}\left(\hat{\boldsymbol{I}}_{s \alpha \beta} \widehat{\boldsymbol{U}}_{s \alpha \beta}\right)
$$

In Formula (9), $\widehat{U}_{s \alpha \beta}$ is the value which lags $U_{s \alpha \beta}$ by 90 electrical degrees. 
The delayed value of stator voltages can be represented as follows:

$$
\left\{\begin{array}{l}
\widehat{u}_{s \alpha}^{+}=\left|\boldsymbol{U}_{s \alpha \beta}^{+}\right| \cos \left(\omega_{s} t+\theta_{u}^{+}-\pi / 2\right)=\left|\boldsymbol{U}_{s \alpha \beta}^{+}\right| \sin \left(\omega_{s} t+\theta_{u}^{+}\right)=u_{s \beta}^{+} \\
\widehat{u}_{s \beta}^{+}=\left|\boldsymbol{U}_{s \alpha \beta}^{+}\right| \sin \left(\omega_{s} t+\theta_{u}^{+}-\pi / 2\right)=-\left|\boldsymbol{U}_{s \alpha \beta}^{+}\right| \cos \left(\omega_{s} t+\theta_{u}^{+}\right)=-u_{s \alpha}^{+} \\
\widehat{u}_{s \alpha}^{-}=\left|\boldsymbol{U}_{s \alpha \beta}^{-}\right| \cos \left(-\omega_{s} t+\theta_{u}^{-}+\pi / 2\right)=-\left|\boldsymbol{U}_{s \alpha \beta}^{-}\right| \sin \left(-\omega_{s} t+\theta_{u}^{-}\right)=-u_{s \beta}^{-} \\
\widehat{u}_{s \beta}^{-}=\left|\boldsymbol{U}_{s \alpha \beta}^{-}\right| \sin \left(-\omega_{s} t+\theta_{u}^{-}+\pi / 2\right)=\left|\boldsymbol{U}_{s \alpha \beta}^{-}\right| \cos \left(-\omega_{s} t+\theta_{u}^{-}\right)=u_{s \alpha}^{-}
\end{array}\right.
$$

According to Formulas (1), (9), and (10), the representations of new active power and traditional active power are consistent when only positive sequence voltage exists. This indicates that the power tracking strategy can still work well via the new active power under balanced grid voltage.

$\boldsymbol{P}_{s n}$ can be then further denoted as follows:

$$
\boldsymbol{P}_{s n}=\boldsymbol{P}_{s n 0}+\boldsymbol{P}_{s n 1}+\boldsymbol{P}_{s n 2}
$$

The equation $\boldsymbol{P}_{s n 0}=\boldsymbol{P}_{s 0}$ can be easily satisfied, and $\boldsymbol{P}_{s n 1}$ and $\boldsymbol{P}_{s n 2}$ are represented as follows:

$$
\left\{\begin{array}{l}
\boldsymbol{P}_{s n 1}=\frac{3}{2}\left(\widehat{u}_{s \alpha}^{+} i_{s \beta}^{-}-\widehat{u}_{s \beta}^{+} i_{s \alpha}^{-}\right)=\frac{3}{2}\left(u_{s \beta}^{+} i_{s \beta}^{-}+u_{s \alpha}^{+} i_{s \alpha}^{-}\right)=\frac{3}{2}\left|U_{s}^{+} \| I_{s}^{-}\right| \cos \left(2 \omega_{s} t+\theta_{u}^{+}-\theta_{i}^{-}\right) \\
\boldsymbol{P}_{s n 2}=\frac{3}{2}\left(\widehat{u}_{s \alpha}^{-} i_{s \beta}^{+}-\widehat{u}_{s \beta}^{-} i_{s \alpha}^{+}\right)=-\frac{3}{2}\left(u_{s \beta}^{-} i_{s \beta}^{+}+u_{s \alpha}^{-} i_{s \alpha}^{+}\right)=-\frac{3}{2}\left|U_{s}^{-}\right|\left|I_{s}^{+}\right| \cos \left(2 \omega_{s} t+\theta_{i}^{+}-\theta_{u}^{-}\right)
\end{array}\right.
$$

According to Formulas (8) and (12):

$$
\left\{\begin{array}{l}
T_{e 1}=\frac{P_{s n 1}}{\omega_{s}} \\
T_{e 2}=\frac{P_{s n 2}}{\omega_{s}}
\end{array}\right.
$$

Because $\theta_{u}^{+}, \theta_{u}^{-}, \theta_{i}^{+}$, and $\theta_{i}^{-}$are the initial phase angles of positive and negative parts of voltage and current, then:

$$
\theta_{i}^{+}-\theta_{u}^{+}=-\left(\theta_{i}^{-}-\theta_{u}^{-}\right)
$$

Then:

$$
\begin{gathered}
\sin \left(\theta_{u}^{+}+\theta_{u}^{-}-\theta_{i}^{+}-\theta_{i}^{-}\right)=\sin \left(2 \omega_{s} t+\theta_{u}^{+}-\theta_{i}^{-}\right) \cos \left(2 \omega_{s} t+\theta_{i}^{+}-\theta_{u}^{-}\right) \\
-\cos \left(2 \omega_{s} t+\theta_{u}^{+}-\theta_{i}^{-}\right) \sin \left(2 \omega_{s} t+\theta_{i}^{+}-\theta_{u}^{-}\right)=0
\end{gathered}
$$

Therefore:

$$
\frac{\cos \left(2 \omega_{s} t+\theta_{i}^{+}-\theta_{u}^{-}\right)}{\sin \left(2 \omega_{s} t+\theta_{i}^{+}-\theta_{u}^{-}\right)}=\frac{\cos \left(2 \omega_{s} t+\theta_{u}^{+}-\theta_{i}^{-}\right)}{\sin \left(2 \omega_{s} t+\theta_{u}^{+}-\theta_{i}^{-}\right)}
$$

$\boldsymbol{P}_{s n 1}+\boldsymbol{P}_{s n 2}=0$ and $\boldsymbol{Q}_{s 1}+\boldsymbol{Q}_{s 2}=0$ can be satisfied simultaneously according to Formulas (6), (12), and (16). Thus, if the reference active power is placed as a constant and to track $\boldsymbol{P}_{s n}$, first, $\boldsymbol{P}_{s n 1}+\boldsymbol{P}_{s n 2}=0$ is satisfied, and then $Q_{s 1}+Q_{s 2}=0$ is established. Thus, a harmonic attenuation for reactive power can be achieved. Moreover, in terms of Formula (13), $T_{e}=T_{e 0}$ is satisfied and electromagnetic torque ripples are restrained.

According to Formula (9), the derivatives of $\boldsymbol{P}_{S}$ and $\boldsymbol{Q}_{s}$ can be represented as follows:

$$
\left\{\begin{array}{l}
\frac{d \boldsymbol{P}_{s}}{d t}=-\frac{3}{2} \operatorname{Im}\left(\frac{d \hat{\boldsymbol{I}}_{s \alpha \beta}}{d t} \widehat{\boldsymbol{U}}_{s \alpha \beta}+\hat{\boldsymbol{I}}_{s \alpha \beta} \frac{d \widehat{\boldsymbol{U}}_{s \alpha \beta}}{d t}\right) \\
\frac{d \boldsymbol{Q}_{s}}{d t}=\frac{3}{2} \operatorname{Im}\left(\frac{d \hat{\boldsymbol{I}}_{s \alpha \beta}}{d t} \boldsymbol{U}_{s \alpha \beta}+\hat{\boldsymbol{I}}_{s \alpha \beta} \frac{d \boldsymbol{U}_{s \alpha \beta}}{d t}\right)
\end{array}\right.
$$


Considering Formula (1) and neglecting the effect of stator resistance and rotor resistance, the derivative of stator current can be deduced as follows:

$$
\frac{d \boldsymbol{I}_{s \alpha \beta}}{d t}=-\frac{1}{\rho L_{m}}\left[\boldsymbol{V}_{r \alpha \beta}-\frac{L_{r}}{L_{m}} \boldsymbol{U}_{s \alpha \beta}+j \omega_{r}\left(-\rho L_{m} \boldsymbol{I}_{s \alpha \beta}+\frac{L_{r}}{L_{m}} \boldsymbol{\psi}_{s \alpha \beta}\right)\right],
$$

where $\rho=\left(L_{r} L_{s} / L_{m}^{2}-1\right)$.

The derivative of stator voltage under stationary reference frame can be denoted as follows:

$$
\begin{gathered}
\frac{d \boldsymbol{U}_{s \alpha \beta}}{d t}=-j \omega_{s}\left|\boldsymbol{U}_{s \alpha \beta}^{+}\right| j e^{j\left(\omega_{s} t+\theta_{u}^{+}\right)}-j \omega_{s}\left|\boldsymbol{U}_{s \alpha \beta}^{-}\right| e^{j\left(-\omega_{1} t+\theta_{u}^{-}\right)}=j \omega_{s} \boldsymbol{U}_{s \alpha \beta}^{+}-j \omega_{1} \boldsymbol{U}_{s \alpha \beta}^{-}=-\omega_{s} \widehat{\boldsymbol{U}}_{s \alpha \beta} \\
\frac{d \widehat{\boldsymbol{U}}_{s \alpha \beta}}{d t}=(-j) j \omega_{s} \boldsymbol{U}_{s \alpha \beta}^{+}+j\left(-j \omega_{1} \boldsymbol{U}_{s \alpha \beta}\right)=\omega_{1} \boldsymbol{U}_{s \alpha \beta}^{+}+\omega_{1} \boldsymbol{U}_{s \alpha \beta}^{-}=\omega_{1} \boldsymbol{U}_{s \alpha \beta}
\end{gathered}
$$

By substituting Formulas (18)-(20) into Formula (17), and converting to the matrix form:

$$
\begin{aligned}
\frac{d}{d t}\left[\begin{array}{c}
\boldsymbol{P}_{s n} \\
Q_{s}
\end{array}\right]= & -\frac{3}{2 \rho L_{m}}\left[\begin{array}{cc}
-\widehat{u}_{s \beta} & \widehat{u}_{s \alpha} \\
u_{s \beta} & -u_{s \alpha}
\end{array}\right]\left[\begin{array}{c}
V_{r \alpha} \\
V_{r \beta}
\end{array}\right]+\frac{3 L_{r}}{2 \rho L_{m}^{2}}\left[\begin{array}{c}
u_{s \beta} \widehat{u}_{s \alpha}-u_{s \alpha} \widehat{u}_{s \beta} \\
0
\end{array}\right] \\
& -\frac{3 L_{r} \omega_{r}}{2 \rho L_{m}^{2}}\left[\begin{array}{c}
\psi_{s \beta} \widehat{u}_{s \alpha}-\psi_{s \alpha} \widehat{u}_{s \beta} \\
\psi_{s \alpha} u_{s \beta}-\psi_{s \beta} u_{s \alpha}
\end{array}\right]+\left[\begin{array}{c}
-\omega_{1} Q_{s}-3 / 2 \omega_{r}\left(\widehat{u}_{s \alpha} i_{s \beta}-\widehat{u}_{s \beta} i_{s \alpha}\right) \\
\omega_{1} \boldsymbol{P}_{s n}+3 / 2 \omega_{r}\left(u_{s \alpha} i_{s \alpha}+u_{s \beta} i_{s \beta}\right)
\end{array}\right]
\end{aligned}
$$

\section{Second-Order Sliding Mode Direct Power Controller Design}

Control tasks for DPC are to track the new active power, $\boldsymbol{P}_{s n}$, and reactive power, $\boldsymbol{Q}_{s}$. The tracking errors are as follows:

$$
\left\{\begin{array}{l}
e_{P}=\boldsymbol{P}_{\text {snref }}-\boldsymbol{P}_{s n} \\
e_{Q}=Q_{\text {sref }}-Q_{s}
\end{array}\right.
$$

For the sake of reducing steady state error and maintaining good dynamic performance, the integral form sliding mode surface can be adopted. Therefore, sliding mode surfaces are designed as follows:

$$
\left\{\begin{array}{l}
\sigma_{P}=e_{P}+k_{P} \int_{0}^{t} e_{P}(\tau) d \tau \\
\sigma_{Q}=e_{Q}+k_{Q} \int_{0}^{t} e_{Q}(\tau) d \tau
\end{array},\right.
$$

where $\boldsymbol{\sigma}=\left[\begin{array}{cc}\sigma_{P} & \sigma_{Q}\end{array}\right]^{T}, k_{P} k_{Q}$ are respectively integral gains of new active power and reactive power.

The first-order time derivative of the sliding mode function is calculated as follows:

$$
\begin{gathered}
\dot{\sigma}=F+G V_{r \alpha \beta} \\
F=-\frac{3 L_{r}}{2 \rho L_{m}^{2}}\left[\begin{array}{c}
u_{s \beta} \widehat{u}_{s \alpha}-u_{s \alpha} \widehat{u}_{s \beta} \\
0
\end{array}\right]+\frac{3 L_{r} \omega_{r}}{2 \rho L_{m}^{2}}\left[\begin{array}{c}
\psi_{s \beta} \widehat{u}_{s \alpha}-\psi_{s \alpha} \widehat{u}_{s \beta} \\
\psi_{s \alpha} u_{s \beta}-\psi_{s \beta} u_{s \alpha}
\end{array}\right]-\left[\begin{array}{c}
-\omega_{1} Q_{s}-3 / 2 \omega_{r}\left(\widehat{u}_{s \alpha} i_{s \beta}-\widehat{u}_{s \beta} i_{s \alpha}\right) \\
\omega_{1} P_{s n}+3 / 2 \omega_{r}\left(u_{s \alpha} i_{s \alpha}+u_{s \beta} i_{s \beta}\right)
\end{array}\right]+\left[\begin{array}{c}
K_{p} e_{p} \\
K_{q} e_{q}
\end{array}\right]+\left[\begin{array}{c}
\dot{P}_{s n r e f} \\
\dot{Q}_{s r e f}
\end{array}\right] \\
G=\frac{3}{2 \rho L_{m}}\left[\begin{array}{cc}
-\widehat{u}_{s \beta} & \widehat{u}_{s \alpha} \\
u_{s \beta} & -u_{s \alpha}
\end{array}\right], V_{r \alpha \beta}=\left[\begin{array}{ll}
V_{r \alpha} & V_{r \beta}
\end{array}\right]^{T} .
\end{gathered}
$$

As most known parts of $F$ and $G$ in Formula (24) are regarded as uncertainties, control chattering can be serious if the constant speed FOSM or the super-twisting SOSM are directly applied to Formula (24). Therefore, the controller is constructed as two parts. Formula (24) is firstly represented as known and unknown parts, as follows:

$$
\begin{aligned}
& \dot{\boldsymbol{\sigma}}=\bar{F}+\Delta_{F}+\left(\bar{G}+\Delta_{G}\right) V_{r \alpha \beta} \\
& =\bar{F}+\bar{G} u_{r \alpha \beta}+\Delta_{F}+\Delta_{G} V_{r \alpha \beta} \\
& =\bar{F}+\bar{G} V_{r \alpha \beta}+\Delta
\end{aligned}
$$


where $\bar{F}, \bar{G}$ are the known part and $\Delta=\left[\begin{array}{ll}\Delta_{1} & \Delta_{2}\end{array}\right]^{T}$ contain uncertainty parameters, measuring errors, unmodeled dynamics, and so on. The value $\Delta$ is related to physical parameters; thus, $\left|\dot{\Delta}_{1}\right| \leq L_{\Delta 1}$, $\left|\dot{\Delta}_{2}\right| \leq L_{\Delta 2}$ are undoubtedly satisfied. $L_{\Delta 1}$ and $L_{\Delta 2}$ are constants.

Feedback control is designed as follows:

$$
V_{r \alpha \beta}=\left[\begin{array}{c}
V_{r \alpha} \\
V_{r \beta}
\end{array}\right]=\bar{G}^{-1}\left(-F+\left[\begin{array}{c}
u_{r \alpha} \\
u_{r \beta}
\end{array}\right]\right)
$$

where $u_{r \alpha}$ and $u_{r \beta}$ are auxiliary control. Then:

$$
\dot{\boldsymbol{\sigma}}=\left[\begin{array}{c}
\dot{\sigma}_{P} \\
\dot{\sigma}_{Q}
\end{array}\right]=\bar{F}+\bar{G} V_{r \alpha \beta}+\Delta=\left[\begin{array}{c}
u_{r \alpha} \\
u_{r \beta}
\end{array}\right]+\Delta,
$$

where $u_{r \alpha}$ and $u_{r \beta}$ are designed based on a super-twisting algorithm [28], as follows:

$$
\begin{gathered}
\left\{\begin{array}{l}
u_{r \alpha}=-\lambda_{r \alpha}\left|\sigma_{P}\right|_{1 / 2} \operatorname{sign}\left(\sigma_{P}\right)+u_{r \alpha v}, \\
\dot{u}_{r \alpha v}=-\gamma_{r \alpha} \operatorname{sign}\left(\sigma_{P}\right)
\end{array},\right. \\
\left\{\begin{array}{l}
u_{r \beta}=-\lambda_{r \beta}\left|\sigma_{Q}\right|_{1 / 2} \operatorname{sign}\left(\sigma_{Q}\right)+u_{r \beta v}, \\
\dot{u}_{r \beta v}=-\gamma_{r \beta} \operatorname{sign}\left(\sigma_{Q}\right)
\end{array}\right.
\end{gathered}
$$

where $\lambda_{r \alpha}, \gamma_{r \alpha}, \lambda_{r \beta}$, and $\gamma_{r \beta}$ are control parameters of the super-twisting SOSM.

Finite time stability can be achieved as long as the SOSM, with respect to $\sigma$, can be established and maintained in finite time. The control parameters $\lambda_{r \alpha}, \gamma_{r \alpha}, \lambda_{r \beta}$, and $\gamma_{r \beta}$ can be chosen according to Reference [39], in which the parameters are required as follows:

$$
\begin{cases}\gamma_{r \alpha}>L_{\Delta 1}, & \lambda_{r \alpha}>\sqrt{\gamma_{r \alpha}+L_{\Delta 1}} \\ \gamma_{r \beta}>L_{\Delta 2}, & \lambda_{r \beta}>\sqrt{\gamma_{r \beta}+L_{\Delta 2}}\end{cases}
$$

The values $L_{\Delta 1}$ and $L_{\Delta 2}$ should be calculated and analyzed according to actual operating environment in the wind turbine system. Yet, the accurate values of $L_{\Delta 1}$ and $L_{\Delta 2}$ are usually difficult to acquire. Thus, it has practical meaning to design the adaptive control parameters $\lambda_{r \alpha}, \gamma_{r \alpha}, \lambda_{r \beta}$, and $\gamma_{r \beta}$.

The next procedure is to construct an adaptive law for $\lambda_{r \alpha}, \gamma_{r \alpha}, \lambda_{r \beta}$, and $\gamma_{r \beta}$, establish SOSM with respect to $\sigma_{P}, \sigma_{Q}$ in finite time, and track $\boldsymbol{P}_{s n}, Q_{s}$. The design procedure of $\lambda_{r \beta} \gamma_{r \beta}$ are similar to $\lambda_{r \alpha} \gamma_{r \alpha}$.

Combining Formulas (27) and (28) and introducing state variable $\sigma_{P v}=\Delta_{1}-\gamma_{r \alpha} \int_{0}^{t} \operatorname{sign}\left(\sigma_{P}\right) d \tau$, then:

$$
\left\{\begin{array}{l}
\dot{\sigma}_{P}=-\lambda_{r \alpha}\left|\sigma_{P}\right|_{1 / 2} \operatorname{sign}\left(\sigma_{P}\right)+\sigma_{P v} \\
\dot{\sigma}_{P v}=-\gamma_{r \alpha} \operatorname{sign}\left(\sigma_{P}\right)+\dot{\Delta}_{1}
\end{array}\right.
$$

To choose vector $\xi^{T}=\left[\operatorname{sign}\left(\sigma_{P}\right)\left|\sigma_{P}\right|_{1 / 2} \quad \sigma_{P v}\right]$, an inequation $\frac{d|x|}{d t}=\dot{x} \operatorname{sign}(x)$ is adopted, then:

$$
\left\{\begin{array}{l}
\dot{\xi}_{1}=\frac{1}{2\left|\sigma_{P}\right|^{1 / 2}}\left(-\lambda_{r \alpha}\left|\sigma_{P}\right|_{1 / 2} \operatorname{sign}\left(\sigma_{P}\right)+\sigma_{P v}\right) \\
\dot{\xi}_{2}=-\gamma_{r \alpha} \operatorname{sign}\left(\sigma_{P}\right)+\dot{\Delta}_{1}
\end{array}\right.
$$

To define $\tilde{\dot{\Delta}}_{1}=\left|\sigma_{P}\right|_{1 / 2} \dot{\Delta}_{1}, \boldsymbol{A}=\left[\begin{array}{cc}-\frac{\lambda_{r \alpha}}{2} & \frac{1}{2} \\ -\gamma_{r \alpha} & 0\end{array}\right], \boldsymbol{B}=\left[\begin{array}{l}0 \\ 1\end{array}\right]$, and $\boldsymbol{C}=\left[\begin{array}{ll}1 & 0\end{array}\right]$, according to Formula (32):

$$
\dot{\xi}=\frac{1}{\left|\sigma_{P}\right|^{1 / 2}}\left(A \xi+B \tilde{\dot{\Delta}_{1}}\right)
$$


Considering the Lyapunov function:

$$
V\left(\xi, \lambda_{r \alpha}, \gamma_{r \alpha}\right)=V_{0}(\xi)+\frac{1}{2 a_{1}}\left(\lambda_{r \alpha}-\lambda_{r \alpha}^{*}\right)^{2}+\frac{1}{2 a_{2}}\left(\gamma_{r \alpha}-\gamma_{r \alpha}^{*}\right)^{2}
$$

where $\lambda_{r \alpha}^{*}$ and $\gamma_{r \alpha}^{*}$ are positive constants; $V_{0}(\xi)=\xi^{T} P \xi ; P=\left[\begin{array}{cc}\frac{m_{P}^{2}+4 \mu_{P}}{2} & -\frac{m_{P}}{2} \\ -\frac{m_{P}}{2} & 1\end{array}\right] ; a_{1}, a_{2}$, and $\mu_{P}$ are positive constants, and $m_{P}$ is an arbitrary constant.

Notice that $\boldsymbol{P}$ is a positive definite symmetric matrix, so then the derivative of $V_{0}(\xi)$ is as follows:

$$
\begin{aligned}
& \dot{V}_{0}(\xi)=2 \dot{\xi}^{T} P \xi=\frac{1}{\left|\sigma_{P}\right|^{1 / 2}}\left(2 \xi^{T} A^{T}+2{\widetilde{\dot{\Delta}_{1}}}_{1} B^{T}\right) P \xi \leq \frac{1}{\left|\sigma_{P}\right|^{1 / 2}}\left(2 \xi^{T} A^{T} P \xi+2 \widetilde{\dot{\Delta}}_{1} B^{T} P \xi+L_{\Delta 1}^{2}\left|\sigma_{P}\right|-\widetilde{\dot{\Delta}}_{1}^{2}\right) \\
& =\frac{1}{\left|\sigma_{P}\right|^{1 / 2}}\left(2 \xi^{T} A^{T} P \xi+2 \widetilde{\dot{\Delta}}_{1} B^{T} P \xi+L_{\Delta 1}^{2} \xi^{T} C^{T} C \xi-\tilde{\dot{\Delta}}_{1}^{2}\right) \\
& \leq \frac{1}{\left|\sigma_{P}\right|^{1 / 2}}\left(2 \xi^{T} A^{T} P \xi+L_{\Delta 1}^{2} \xi^{T} C^{T} C \xi+\xi^{T} P B B^{T} P \xi\right) \\
& =\frac{1}{\left|\sigma_{P}\right|^{1 / 2}} \xi^{T}\left(A^{T} P+P A^{T}+L_{\Delta 1}^{2} C^{T} C+P B B^{T} P\right) \xi
\end{aligned}
$$

To define $Q=-\left(A^{T} P+P A^{T}+L_{\Delta 1}^{2} C^{T} C+P B B^{T} P\right)$, Formula (35) can be written as follows:

$$
V_{0}(\xi) \leq-\frac{1}{\left|\sigma_{p}\right|^{1 / 2}} \xi^{T} Q \xi
$$

$A, B, C, P$ are substituted in $Q$, then:

$$
Q=\left[\begin{array}{cc}
2 \lambda_{r \alpha} \mu_{P}+\frac{\lambda_{r \alpha} m_{P}^{2}}{2}-\gamma_{r \alpha} m_{P}-L_{\Delta 1}^{2}-\frac{m_{P}^{2}}{4} & -\mu_{P}-\frac{m_{P}^{2}}{4}-\frac{\lambda_{r \alpha} m_{P}}{4}+\gamma_{r \alpha}+\frac{m_{P}}{2} \\
-\mu_{P}-\frac{m_{P}^{2}}{4}-\frac{\lambda_{r \alpha} m_{P}}{4}+\gamma_{r \alpha}+\frac{m_{P}}{2} & \frac{m_{P}}{2}-1
\end{array}\right]
$$

In order to guarantee positive definiteness, define:

$$
\gamma_{r \alpha}=\mu_{P}+\frac{m_{P}^{2}}{4}+\frac{\lambda_{r \alpha} m_{P}}{4}
$$

Formula (37) is substituted into Formula (38), then:

$$
Q-\frac{m_{P} \boldsymbol{I}}{4}=\left[\begin{array}{cc}
2 \lambda_{r \alpha} \mu_{P}+\frac{\lambda_{r \alpha} m_{P}^{2}}{2}-\mu_{P} m_{P}-\frac{m_{P}^{3}}{4}-L_{\Delta 1}^{2}-\frac{m_{P}^{2}}{4}-\frac{m_{P}}{4} & \frac{m_{P}}{2} \\
\frac{m_{P}}{4}-1
\end{array}\right]
$$

According to properties of the Schur complement, the conditions to guarantee a positive definiteness of $Q$ and a minimum eigenvalue $\lambda_{\min }(Q)>\frac{m_{P}}{4}$ are as follows:

$$
\left\{\begin{array}{l}
\lambda_{r \alpha}>\frac{\frac{m_{P}^{2}}{4}+\left(\mu_{P} m_{P}+\frac{m_{P}^{3}}{4}+L_{\Delta 1}^{2}+\frac{m_{P}^{2}}{4}+\frac{m_{P}}{4}\right)\left(\frac{m_{P}}{4}-1\right)}{\left(2 \mu_{P}+\frac{m_{P}^{2}}{4}\right)\left(\frac{m_{P}}{4}-1\right)} \\
m_{P}>4
\end{array}\right.
$$

According to Formula (36):

$$
V_{0}(\xi) \leq-\frac{1}{\left|\sigma_{P}\right|^{1 / 2}} \xi^{T} Q \xi \leq-\frac{m_{P}}{4\left|\xi_{1}\right|} \xi^{T} \xi=-\frac{m_{P}}{4\left|\xi_{1}\right|}\|\xi\|_{2}=-\frac{m_{P}\|\xi\|}{4\left|\xi_{1}\right|}\|\xi\|
$$

According to $\|\xi\|_{2}^{2}=\xi_{1}^{2}+\xi_{2}^{2}=\left|\boldsymbol{\sigma}_{P}\right|+\xi_{2}^{2}$, then:

$$
\|\xi\|_{2} \geq\left|\xi_{1}\right|
$$


Then, Formula (41) can be represented as follows:

$$
V_{0}(\xi) \leq-\frac{m_{P}}{4}\|\xi\|_{2}
$$

According to positive definite quadratic form $V_{0}(\xi)=\xi^{T} P \xi$ :

$$
\lambda_{\min }(\boldsymbol{P})\|\xi\|_{2}^{2} \leq V_{0}(\xi)=\xi^{T} P \xi \leq \lambda_{\max }(\boldsymbol{P})\|\xi\|_{2}^{2}
$$

In view of Formula (44):

$$
\left(\frac{V_{0}(\xi)}{\lambda_{\max }(\boldsymbol{P})}\right)^{1 / 2} \leq\|\xi\|_{2}
$$

Considering Formulas (43) and (45):

$$
V_{0}(\xi) \leq-r V_{0}^{1 / 2}(\xi)
$$

where $r=\frac{m_{P}}{4 \lambda_{\max }^{1 / 2}(\boldsymbol{P})}$, then:

$$
\begin{aligned}
& \dot{V}\left(\xi, \lambda_{r \alpha}, \gamma_{r \alpha}\right)=-r V_{0}^{1 / 2}(\xi)+\frac{1}{a_{1}}\left(\lambda_{r \alpha}-\lambda_{r \alpha}^{*}\right) \dot{\lambda}_{r \alpha}+\frac{1}{a_{2}}\left(\gamma_{r \alpha}-\gamma_{r \alpha}^{*}\right) \dot{\gamma}_{r \alpha} \\
& =-r V_{0}^{1 / 2}(\xi)-\frac{\beta_{P 1}}{\sqrt{2 a_{1}}}\left|\lambda_{r \alpha}-\lambda_{r \alpha}^{*}\right|-\frac{\beta_{P 2}}{\sqrt{2 a_{2}}}\left|\gamma_{r \alpha}-\gamma_{r \alpha}^{*}\right|+\frac{1}{a_{1}}\left(\lambda_{r \alpha}-\lambda_{r \alpha}^{*}\right) \dot{\lambda}_{r \alpha}+\frac{1}{a_{2}}\left(\gamma_{r \alpha}-\gamma_{r \alpha}^{*}\right) \dot{\gamma}_{r \alpha} \\
& +\frac{\beta_{P 1}}{\sqrt{2 a_{1}}}\left|\lambda_{r \alpha}-\lambda_{r \alpha}^{*}\right|+\frac{\beta P 2}{\sqrt{2 a_{2}}}\left|\gamma_{r \alpha}-\gamma_{r \alpha}^{*}\right| \\
& \leq-\min \left(r, a_{1}, a_{2}\right)\left(\left(V_{0}(\xi)+\frac{1}{2 a_{1}}\left(\lambda_{r \alpha}-\lambda_{r \alpha}^{*}\right)^{2}+\frac{1}{2 a_{2}}\left(\gamma_{r \alpha}-\gamma_{r \alpha}^{*}\right)^{2}\right)^{1 / 2}+\frac{1}{a_{1}}\left(\lambda_{r \alpha}-\lambda_{r \alpha}^{*}\right) \dot{\lambda}_{r \alpha}\right. \\
& +\frac{1}{a_{2}}\left(\gamma_{r \alpha}-\gamma_{r \alpha}^{*}\right) \dot{\gamma}_{r \alpha}+\frac{\beta_{P 1}}{\sqrt{2 a_{1}}}\left|\lambda_{r \alpha}-\lambda_{r \alpha}^{*}\right|+\frac{\beta_{P 2}}{\sqrt{2 a_{2}}}\left|\gamma_{r \alpha}-\gamma_{r \alpha}^{*}\right|
\end{aligned}
$$

where $\beta_{P 1}$ and $\beta_{P 2}$ are positive constants.

The values $\lambda_{r \alpha}$ and $\gamma_{r \alpha}$ are both bounded. Therefore, Formula (47) is written as follows:

$$
\dot{V}\left(\xi, \lambda_{r \alpha}, \gamma_{r \alpha}\right) \leq-\min \left(r, a_{1}, a_{2}\right) V^{1 / 2}+\zeta,
$$

where $\zeta=-\left|\lambda_{r \alpha}-\lambda_{r \alpha}^{*}\right|\left(\frac{1}{a_{1}} \dot{\lambda}_{r \alpha}-\frac{\beta_{P 1}}{\sqrt{2 a_{1}}}\right)-\left|\gamma_{r \alpha}-\gamma_{r \alpha}^{*}\right|\left(\frac{1}{a_{2}} \dot{\gamma}_{r \alpha}-\frac{\beta_{P 2}}{\sqrt{2 a_{2}}}\right)$.

In order to achieve finite time convergence, to make $\zeta=0$, then the adaptive law for $\lambda_{r \alpha}$ and $\gamma_{r \alpha}$ are as follows:

$$
\left\{\begin{array}{l}
\dot{\lambda}_{r \alpha}=\beta_{P 1} \sqrt{\frac{a_{1}}{2}} \\
\dot{\gamma}_{r \alpha}=\beta_{P 2} \sqrt{\frac{a_{2}}{2}}
\end{array}\right.
$$

For the sake of the uniformity of Formulas (49) and (38), choose:

$$
m_{P}=\frac{4 \beta_{P 2}}{\beta_{P 1}} \sqrt{\frac{a_{2}}{a_{1}}}
$$

Formula (48) is rewritten as follows:

$$
\dot{V}\left(\xi, \lambda_{r \alpha}, \gamma_{r \alpha}\right) \leq-\min \left(r, a_{1}, a_{2}\right) V^{1 / 2}
$$

Thus, $V\left(\xi, \lambda_{r \alpha}, \gamma_{r \alpha}\right)$ can converge to zero in finite time, and it can be observed that $V_{0}(\xi)$ can also converge to zero in finite time. Hence, when control gains $\lambda_{r \alpha}$ and $\gamma_{r \alpha}$ are chosen as follows:

$$
\left\{\begin{array}{l}
\dot{\lambda}_{r \alpha}= \begin{cases}\beta_{P 1} \sqrt{\frac{a_{1}}{2}} & \lambda_{r \alpha} \neq 0 \\
0 & \lambda_{r \alpha}=0\end{cases} \\
\gamma_{r \alpha}=\mu_{P}+\frac{m_{P}^{2}}{4}+\frac{\lambda_{r \alpha} m_{P}}{4}
\end{array}\right.
$$


the values $\sigma_{P}$ and $\dot{\sigma}_{P}$ can converge to zero in finite time and SOSM with respect to $\sigma_{P}$ can be established. Then, active power tracking is achieved.

Similarly, when control gains $\lambda_{r \beta}$ and $\gamma_{r \beta}$ are designed as follows:

$$
\left\{\begin{array}{l}
\dot{\lambda}_{r \beta}= \begin{cases}\beta_{Q 1} \sqrt{\frac{a_{3}}{2}} & \lambda_{r \beta} \neq 0 \\
0 & \lambda_{r \beta}=0\end{cases} \\
\gamma_{r \beta}=\mu_{Q}+\frac{m_{Q}^{2}}{4}+\frac{\lambda_{r \beta} m_{Q}}{4}
\end{array}\right.
$$

SOSM with respect to $\sigma_{Q}$ can be established in finite time and the reactive power tracking objective can be achieved.

\section{Simulation Results}

\subsection{Control System Overview}

The system control diagram can be described as in Figure 2 according to the aforementioned design procedure.

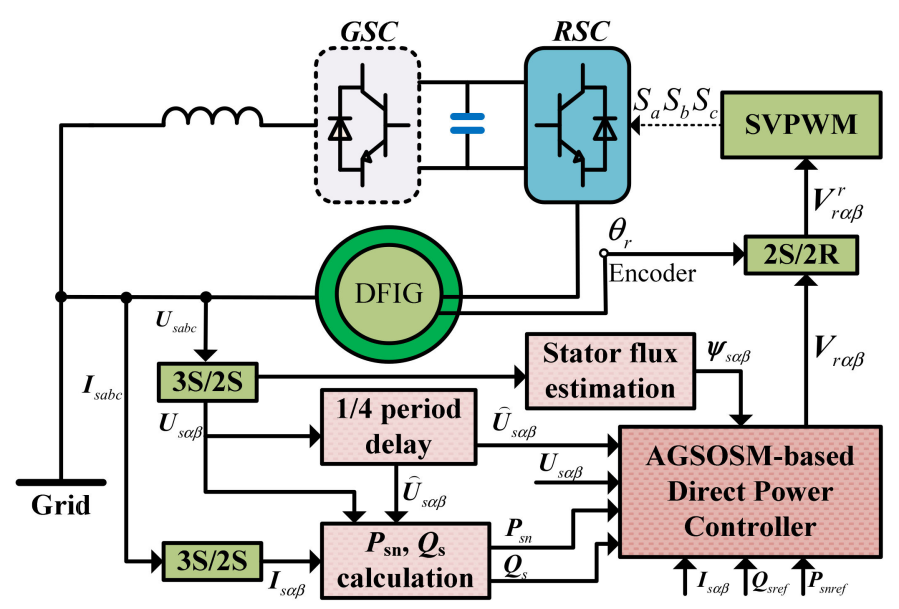

Figure 2. System control diagram.

Firstly, the measured three-phase stator voltage, $\boldsymbol{U}_{s a b c}$, and current, $\boldsymbol{I}_{s a b c}$, are converted to $\boldsymbol{U}_{s \alpha \beta}$ and $\boldsymbol{I}_{s \alpha \beta}$ under two phase stationary frame, and $\widehat{\boldsymbol{U}}_{s \alpha \beta}$ is calculated via $\boldsymbol{U}_{s \alpha \beta}$. Secondly, $\boldsymbol{\psi}_{s \alpha \beta}$ is estimated according to stator voltage, and the active power, $\boldsymbol{P}_{s n}$, and reactive power, $\boldsymbol{Q}_{s}$, are calculated by Formulas (1) and (9). Furthermore, the reference values of power, stator voltage, stator flux linkage, and stator current are applied as inputs of the AGSOSM direct power controller. Then, $\boldsymbol{V}_{r \alpha \beta}$ can be obtained via related controller Formulas (26), (28), (29), (52), and (53). Then, $V_{r \alpha \beta}$ is converted to $V_{r \alpha \beta}^{r}$ under the rotor reference frame. Finally, space vector pulse width modulation (SVPWM) is controlled by $V_{r \alpha \beta}^{r}$ to generate $S_{a}, S_{b}$, and $S_{c}$. It is observed that the control system is rather simple, and phase sequence, PLL, and the upper bounds of the uncertainties are not needed.

It should be noted that, though stator flux linkage is sometimes calculated via $\psi_{s \alpha \beta}=\int_{0}^{t}\left[\boldsymbol{U}_{s \alpha \beta}-R_{s} \boldsymbol{I}_{s \alpha \beta}\right]$, an integral operator is usually substituted by band-pass filter to avoid the drift phenomenon. A band-pass filter can help filter static state stator flux, which is a component that appears on account of instantaneous voltage dips, since the stator flux cannot change suddenly. The natural flux gives rise to a large voltage in the rotor and may induce an out of control state for the electric generator. Thus, stator flux linkage is expressed as follows:

$$
\psi_{s \alpha \beta}(p)=\frac{p}{\left(p+\omega_{c}\right)^{2}} \boldsymbol{U}_{s \alpha \beta}(p)
$$


where $p$ is a Laplace operator and $\omega_{c}$ is the cut-off frequency of the filter.

\subsection{Simulation Experiment}

Simulations were carried out under the MATLAB/Simulink platform for a 2MW DFIG with the characteristics shown in Table 1. In order to verify the performance of the proposed AGSOSM-DPC strategy, comparative simulations based on FOSM-DPC [22] and FOSM-EDPC [25] were also executed. The dc-link voltage was maintained as $1200 \mathrm{~V}$ via a method mentioned in Reference [22], which is not included here. The sampling frequency was set as $4 \mathrm{kHz}$ for both the control strategies. The controller parameters were $k_{P}=3500, k_{Q}=3500, \beta_{P 1}=5.7, a_{1}=3.5, \mu_{P}=6.5, m_{P}=2.1, \beta_{Q 1}=4.5, a_{3}=2.2$, $\mu_{Q}=6.2$, and $m_{Q}=3.5$.

Table 1. DFIG parameters.

\begin{tabular}{ll}
\hline Rated Power $(\mathrm{MW})$ & 2 \\
Line-to-line voltage $(\mathrm{rms})(\mathrm{V})$ & 690 \\
Stator frequency $(\mathrm{Hz})$ & 50 \\
Stator-to-rotor ratio & 3 \\
Rs (ohm) & 0.001518 \\
$\mathrm{Rr}(\mathrm{ohm})$ & 0.002087 \\
$\mathrm{Ls}(\mathrm{mH})$ & 0.059906 \\
$\mathrm{Lr}(\mathrm{mH})$ & 0.08206 \\
$\mathrm{Lm}(\mathrm{mH})$ & 2.4 \\
Pole pairs & 2 \\
Lumped inertia constant $\left(\mathrm{kg} \cdot \mathrm{m}^{2}\right)$ & 17.23 \\
\hline
\end{tabular}

Figures 3-5 show the variations of active power, reactive power, electromagnetic torque, stator current, and rotor current when active power reference changed from $1 \mathrm{MW}$ to $2 \mathrm{MW}$, and reactive power changed from 1 MVar to 0 MVar under balanced grid voltage for the three control strategies. The response curves demonstrate excellent steady state and dynamic characteristics under all the three control strategies. Table 2 shows a quantitative comparison of the transitory response and power ripples of active power and reactive power, and total harmonic distortion (THD) of stator current and rotor current. It is evident that better dynamic performance is achieved under the proposed control strategy.

Table 2. Quantitative comparison under the three control strategies.

\begin{tabular}{cccccccc}
\hline \multirow{2}{*}{ Control Strategy } & \multicolumn{2}{c}{$\begin{array}{c}\text { Transitory } \mathbf{r} \\
\text { Response } \mathbf{( m s )}\end{array}$} & \multicolumn{2}{c}{ Power Ripple (\%) } & \multicolumn{2}{c}{ THD (\%) } \\
\cline { 2 - 8 } & $\boldsymbol{P}$ & $\boldsymbol{Q}$ & $\boldsymbol{P}$ & $\boldsymbol{Q}$ & $\mathbf{I s}$ & $\mathbf{I r}$ \\
\hline AGSOSM-DPC & 1.3 & 1.6 & 12.7 & 17.4 & 1.9 & 2.7 \\
FOSM-EDPC & 1.3 & 1.7 & 15.3 & 19.7 & 2.3 & 3.2 \\
FOSM-DPC & 1.5 & 2.1 & 19.5 & 21.2 & 5.2 & 6.1 \\
\hline
\end{tabular}



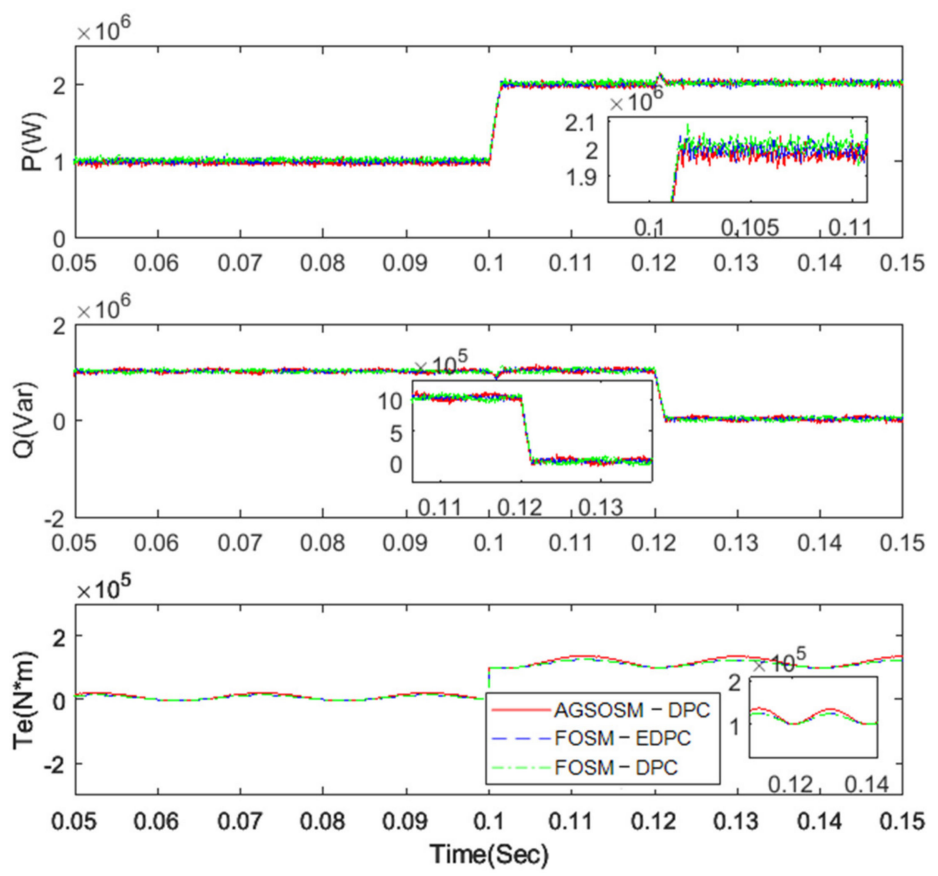

Figure 3. Active power, reactive power, and electromagnetic torque under balanced grid voltage.
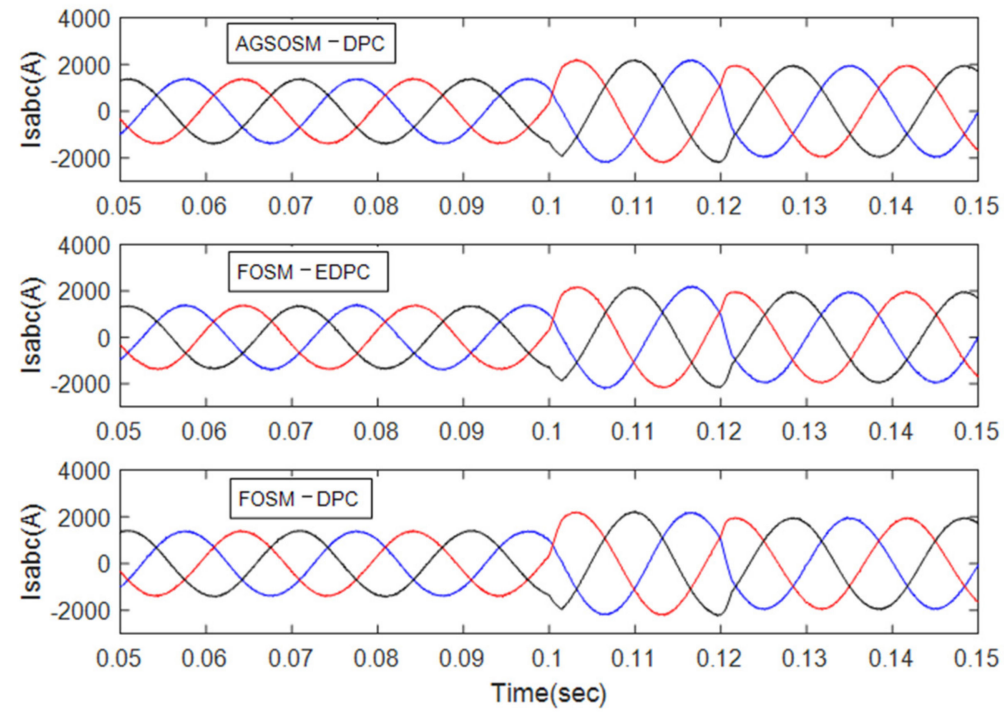

Figure 4. Stator current under balanced grid voltage. 

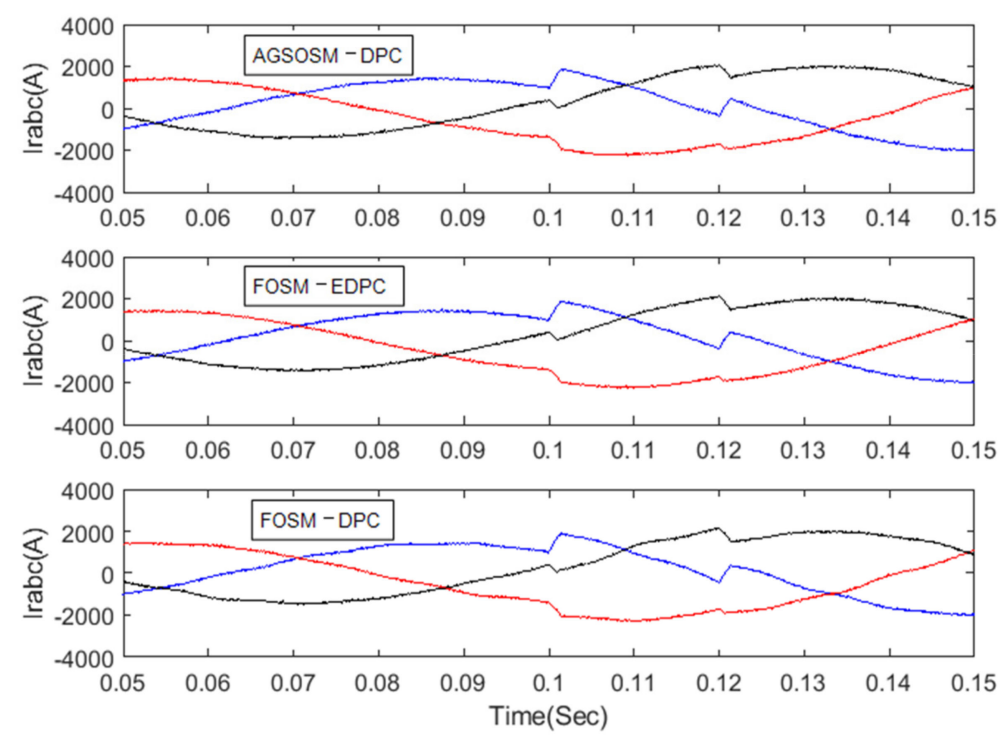

Figure 5. Rotor current under balanced grid voltage.

Figure 6 shows control voltages under the three control strategies. It can be observed that control action is continuous because the SOSM method is adopted in the proposed AGSOSM control strategy. The control chattering is smaller, which means a longer service life. Figure 7 displays control parameters for the proposed AGSOSM control law. The control parameters can be adaptively adjusted according to system variation.
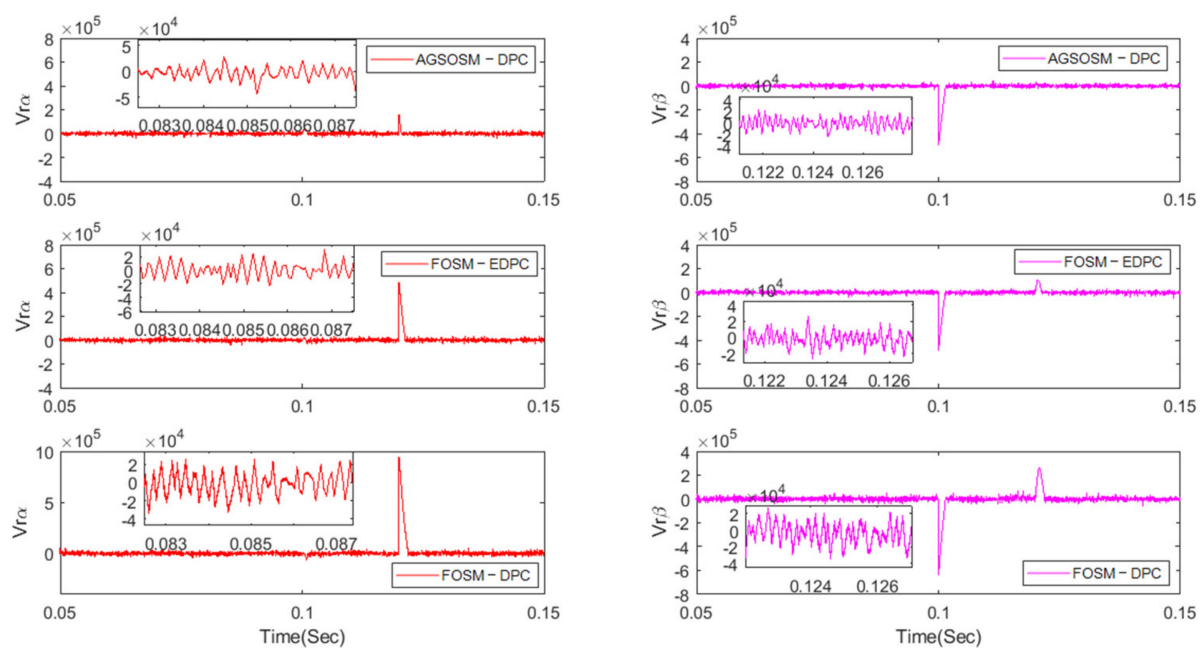

Figure 6. Control voltage under two phase stationary frame under balanced grid voltage. 

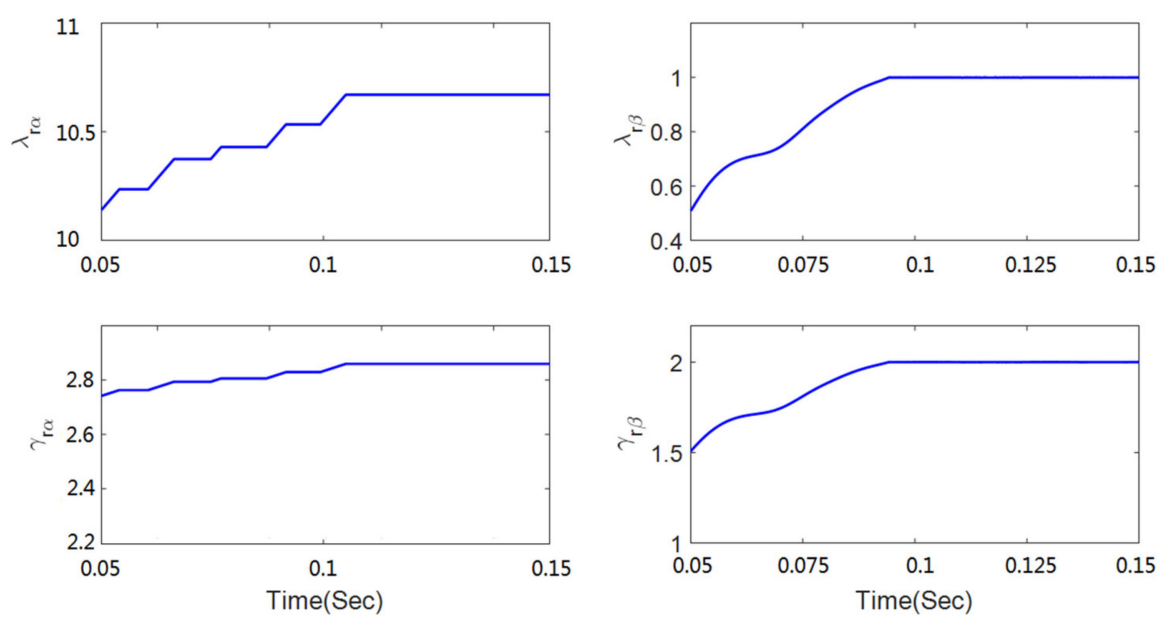

Figure 7. Control parameters for the proposed AGSOSM control law.

Steady state responses under unbalanced grid voltage for the three control strategies are shown in Figures 8-10. Active and reactive power are respectively set as $2 \mathrm{MW}$ and 0.5MVAr. Under the FOSM-DPC scheme, though active power and reactive power are maintained as the reference values, electromagnetic torque ripples are bigger and the stator current contains more harmonic components than the other two schemes. For the FOSM-EDPC and AGSOSM-DPC schemes, active power contains more ripples because the new active power is selected as the control target to obtain sinusoidal stator currents. As is shown in Figure 11, control chattering is smaller under the proposed AGSOSM-DPC scheme than that under the other two control schemes.
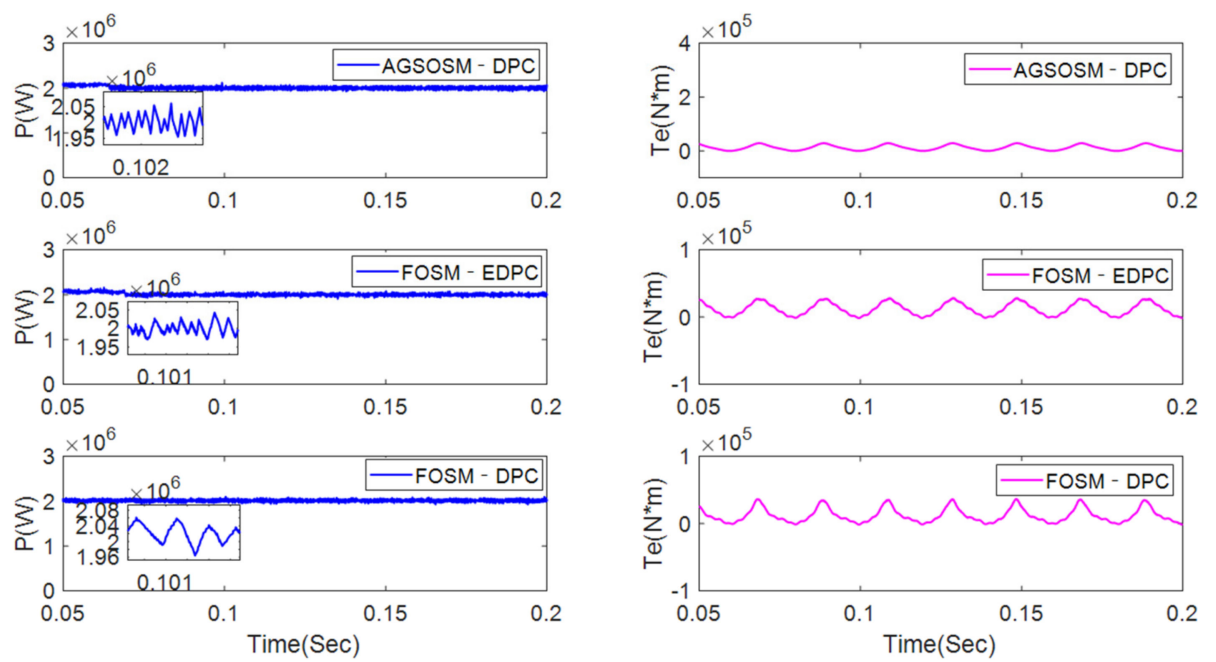

Figure 8. Active power and electromagnetic torque under unbalanced grid voltage. 

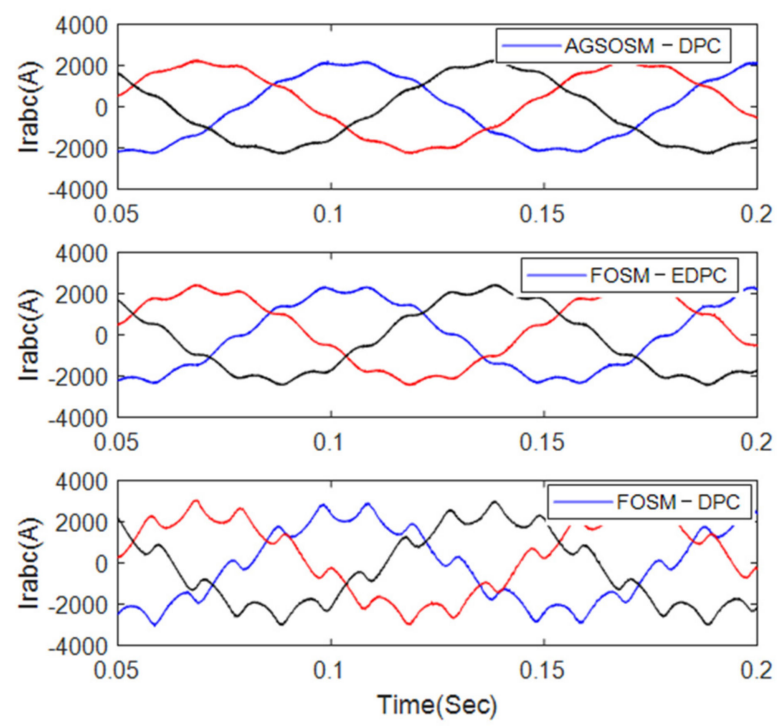

Figure 9. Rotor current under unbalanced grid voltage.
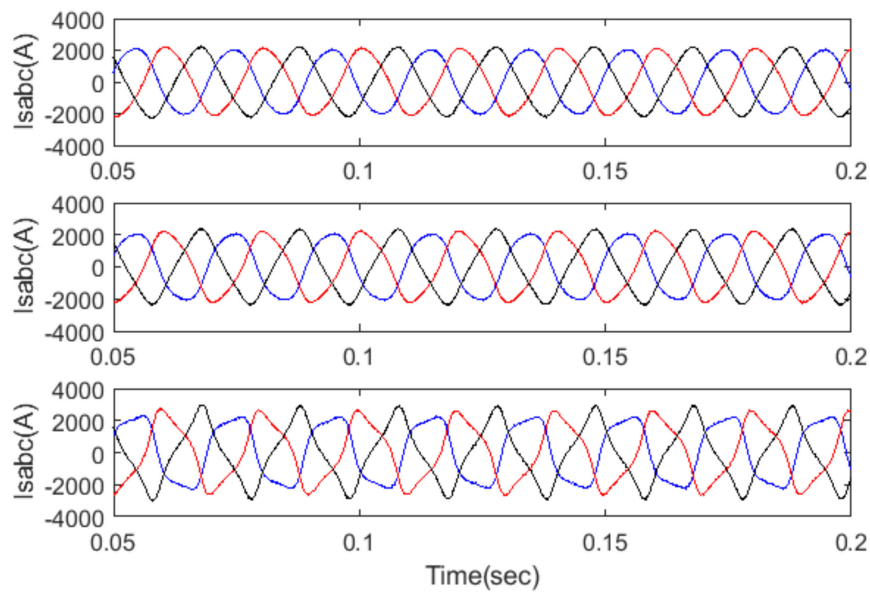

Figure 10. Stator current under unbalanced grid voltage.
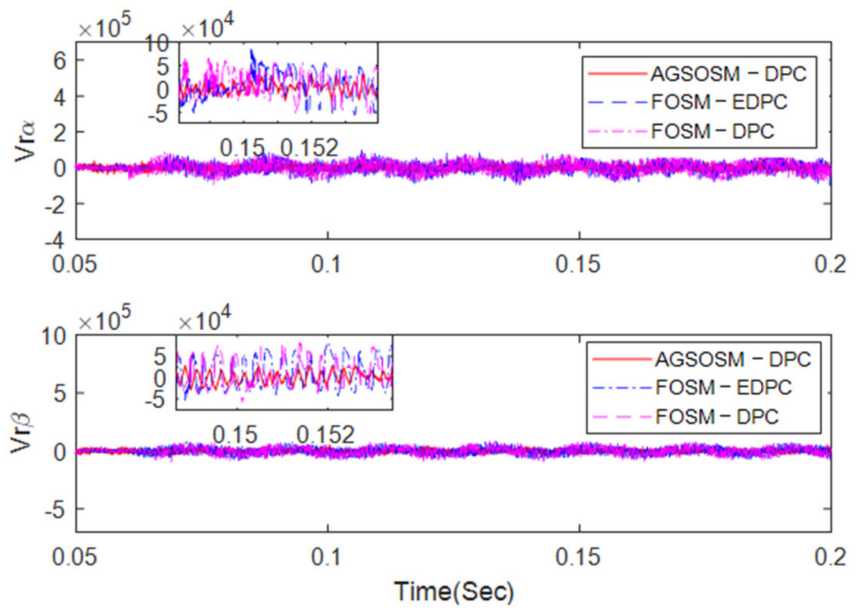

Figure 11. Control voltage under the proposed AGSOSM-DPC strategy. 
To expediently verify the robustness of the proposed AGSOSM-DPC scheme, the uncertainty of mutual inductance, which is often influenced by stator and rotor cores, is taken into account. In addition to this, the variations of stator resistance and rotor resistance should also be specially considered. The mutual inductance, stator resistance, and rotor resistance are reduced and increased to $50 \% L_{m}, 50 \%$ Rs, 50\% $R_{\mathrm{r}}$ and $120 \% L_{m}, 120 \%$ Rs, and $120 \% R_{\mathrm{r}}$, respectively. The stator current, active power, and electromagnetic torque are shown in Figure 12, which displays that the relative responses are almost the same as that of Figures 8 and 10. This means the system is robust under the proposed AGSOSM-DPC.
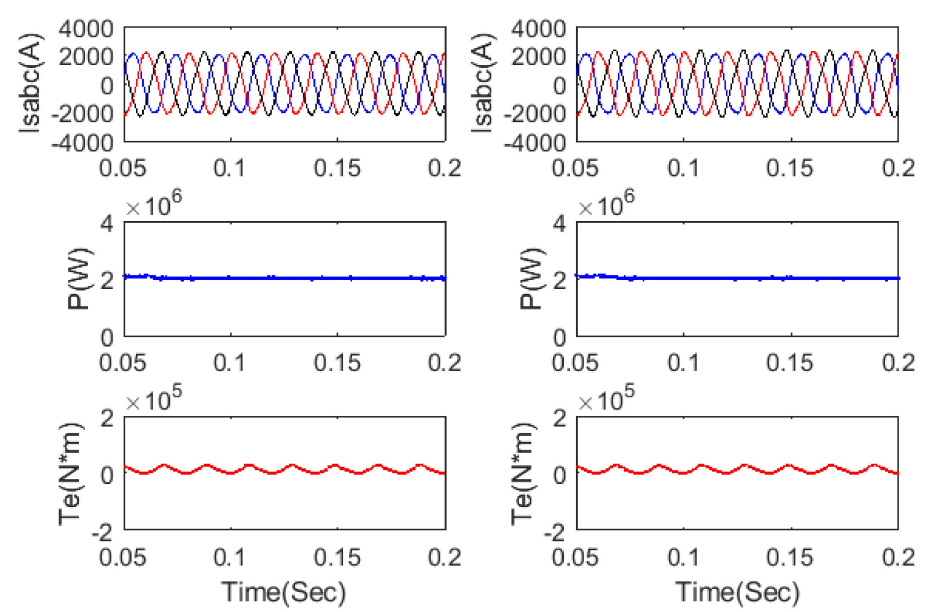

Figure 12. Responses with $50 \% L_{m}, 50 \% R s, 50 \% R_{r}$ and $120 \% L_{m}, 120 \%$ Rs, and $120 \% R_{\mathrm{r}}$.

\section{Conclusions}

This study proposes a novel DPC scheme for a wind-turbine-driven DFIG based on an AGSOSM super-twisting algorithm. First, SOSM direct power controllers were designed based on the detailed analysis for a DFIG model under a two-phase stationary reference frame. Then, adaptive control gains were constructed considering the unknown upper bound of uncertainty. The simulation results indicate that the proposed scheme is valid for balanced and unbalanced grid voltage. Remarkable steady-state performance and dynamic performance can be achieved under balanced grid voltage, and control chattering is significantly reduced. Under unbalanced grid voltage, electromagnetic torque ripples are restrained, and stator currents are sinusoidal. They can be simultaneously achieved without PLL and phase sequence decomposition. The more important contributions are that severe control chattering is significantly reduced, and the upper bound of uncertainty is not necessary during the operational process.

Author Contributions: Conceptualization, Y.H.; Funding acquisition, Y.H.; Methodology, Y.H.; Software, R.M.; Validation, Y.H. and R.M.; Writing—original draft, Y.H.; Writing—review \& editing, R.M.

Funding: This work was funded by National Natural Science Foundation of China under Grant 61803230, 61773015 and 61803154; A Project of Shandong Province Higher Educational Science and Technology Program under Grant J18KA330;University outstanding youth innovation team development plan of Shandong Province under Grant 2019KJN023; Key research and development plan project of Shandong Province under Grant 2018GGX105003.

Conflicts of Interest: The authors declare no conflict of interest.

\section{Nomenclature}
$U_{s}, V_{r} \quad$ Stator, rotor voltage vectors.
$I_{S}, I_{r} \quad$ Stator, rotor current vectors.
$Q_{s}, Q_{r} \quad$ Stator output active and reactive powers.
$\psi_{S}, \psi_{r} \quad$ Stator, rotor flux linkage vectors.
$\boldsymbol{R}_{S}, R_{r} \quad$ Stator, rotor resistances
$L_{m}, L_{s}, L_{r} \quad$ Mutual inductance, Stator, rotor self-inductances.
$\alpha, \beta \quad$ Stator $\alpha, \beta$ axis 


\section{References}

1. Djilali, L.; Sanchez, E.N.; Belkheiri, M. Real-time neural sliding mode field oriented control for a DFIG-based wind turbine under balanced and unbalanced grid conditions. IET Renew. Power Gener. 2019, 13, 618-632. [CrossRef]

2. Available online: https://www.irena.org/ (accessed on 11 August 2019).

3. Liu, Y.; Wang, Z.; Xiong, L.; Wang, J.; Jiang, X.; Bai, G.; Li, R.; Liu, S. DFIG wind turbine sliding mode control with exponential reaching law under variable wind speed. Int. J. Electr. Power Energy Syst. 2018, 96, 253-260. [CrossRef]

4. Xiong, L.; Li, P.; Wu, F.; Ma, M.; Khan, M.W.; Wang, J. A coordinated high-order sliding mode control of DFIG wind turbine for power optimization and grid synchronization. Int. J. Electr. Power Energy Syst. 2019, 105, 679-689. [CrossRef]

5. Merabet, A.; Eshaft, H.; Tanvir, A.A. Power-current controller based sliding mode control for DFIG-wind energy conversion system. IET Renew. Power Gener. 2018, 12, 1155-1163. [CrossRef]

6. Yuan, T.; Wang, J.; Guan, Y.; Liu, Z.; Song, X.; Che, Y.; Cao, W. Virtual Inertia Adaptive Control of a Doubly Fed Induction Generator (DFIG) Wind Power System with Hydrogen Energy Storage. Energies 2018, 11, 904. [CrossRef]

7. Villanueva, I.; Rosales, A.; Ponce, P.; Molina, A. Grid-voltage-oriented sliding mode control for DFIG under balanced and unbalanced grid Faults. IEEE Trans. Sustain. Energy 2018, 9, 1090-1098. [CrossRef]

8. Nian, H.; Cheng, P.; Zhu, Z.Q. Coordinated direct power control of DFIG system without phase-locked loop under unbalanced grid voltage conditions. IEEE Trans. Power Electron. 2015, 31, 2905-2918. [CrossRef]

9. Cheng, P.; Nian, H.; Wu, C.; Zhu, Z.Q. Direct stator current vector control strategy of DFIG without phase-locked loop during network unbalance. IEEE Trans. Power Electron. 2016, 32, 284-297. [CrossRef]

10. Ayyarao, T.S.L.V. Modified vector controlled DFIG wind energy system based on barrier function adaptive sliding mode control. Prot. Control Mod. Power Syst. 2019, 4, 4. [CrossRef]

11. Mehdipour, C.; Hajizadeh, A.; Mehdipour, I. Dynamic modeling and control of DFIG-based wind turbines under balanced network conditions. Int. J. Electr. Power Energy Syst. 2016, 83, 560-569. [CrossRef]

12. Bektache, A.; Boukhezzar, B. Nonlinear predictive control of a DFIG-based wind turbine for power capture optimization. Int. J. Electr. Power Energy Syst. 2018, 101, 92-102. [CrossRef]

13. Alsmadi, Y.M.; Xu, L.; Blaabjerg, F.; Ortega, A.J.; Abdelaziz, A.Y.; Wang, A.; Albataineh, Z. Detailed investigation and performance improvement of the dynamic behavior of grid-connected DFIG-based wind turbines under LVRT conditions. IEEE Trans. Ind. Appl. 2018, 54, 4795-4812. [CrossRef]

14. Kashkooli, M.R.A.; Madani, S.M.; Lipo, T.A. Improved Direct Torque Control for a DFIG under Symmetrical Voltage Dip with Transient Flux Damping. IEEE Trans. Ind. Electron. 2019. [CrossRef]

15. Xu, L.; Cartwright, P. Direct active and reactive power control of DFIG for wind energy generation. IEEE Trans. Energy Conver. 2006, 21, 750-758. [CrossRef]

16. Zandzadeh, M.J.; Vahedi, A. Modeling and improvement of direct power control of DFIG under unbalanced grid voltage condition. Int. J. Electr. Power Energy Syst. 2014, 59, 58-65. [CrossRef]

17. Debouza, M.; Al-Durra, A.; Errouissi, R.; Muyeen, S.M. Direct power control for grid-connected doubly fed induction generator using disturbance observer based control. Renew. Energy 2018, 125, 365-372. [CrossRef]

18. Zhang, Y.; Jiao, J.; Xu, D. Direct Power Control of Doubly Fed Induction Generator Using Extended Power Theory Under Unbalanced Network. IEEE Trans. Power Electron. 2019. [CrossRef]

19. Benamor, A.; Benchouia, M.T.; Srairi, K.; Benbouzid, M.E. A novel rooted tree optimization apply in the high order sliding mode control using super-twisting algorithm based on DTC scheme for DFIG. Int. J. Electr. Power Energy Syst. 2019, 108, 293-302. [CrossRef]

20. Han, Y.; Ma, R.; Cui, J. Adaptive higher-order sliding mode control for islanding and grid-connected operation of a microgrid. Energies 2018, 11, 1459. [CrossRef]

21. Mystkowski, A. Lyapunov sliding-mode observers with application for active magnetic bearing operated with zero-bias flux. J. Dyn. Sys. Meas. Control 2019, 141, 041006. [CrossRef]

22. Hu, J.; Nian, H.; Hu, B.; He, Y.; Zhu, Z.Q. Direct active and reactive power regulation of DFIG using sliding-mode control approach. IEEE Trans. Energy Convers. 2010, 25, 1028-1039. [CrossRef] 
23. Shang, L.; Hu, J. Sliding-mode-based direct power control of grid-connected wind-turbine-driven doubly fed induction generators under unbalanced grid voltage conditions. IEEE Trans. Energy Convers. 2012, 27, 362-373. [CrossRef]

24. Li, S.; Wang, H.; Tian, Y.; Aitouch, A.; Klein, J. Direct power control of DFIG wind turbine systems based on an intelligent proportional-integral sliding mode control. ISA Trans. 2016, 64, 431-439. [CrossRef] [PubMed]

25. Sun, D.; Wang, X.; Nian, H.; Zhu, Z.Q. A sliding-mode direct power control strategy for DFIG under both balanced and unbalanced grid conditions using extended active power. IEEE Trans. Power Electron. 2017, 33, 1313-1322. [CrossRef]

26. Martinez, M.I.; Susperregui, A.; Tapia, G.; Xu, L. Sliding-mode control of a wind turbine-driven double-fed induction generator under non-ideal grid voltages. IET Renew. Power Gener. 2013, 7, 370-379. [CrossRef]

27. Martinez, M.I.; Tapia, G.; Susperregui, A.; Camblong, H. Sliding-mode control for DFIG rotor-and grid-side converters under unbalanced and harmonically distorted grid voltage. IEEE Trans. Energy Convers. 2012, 27, 328-339. [CrossRef]

28. Liu, X.; Han, Y.; Wang, C. Second-order sliding mode control for power optimisation of DFIG-based variable speed wind turbine. IET Renew. Power Gener. 2016, 11, 408-418. [CrossRef]

29. Behera, A.K.; Chalanga, A.; Bandyopadhyay, B. A new geometric proof of super-twisting control with actuator saturation. Automatica 2018, 87, 437-441. [CrossRef]

30. Beltran, B.; Benbouzid, M.E.H.; Ahmed-Ali, T. Second-order sliding mode control of a doubly fed induction generator driven wind turbine. IEEE Trans. Energy Convers. 2012, 27, 261-269. [CrossRef]

31. Benbouzid, M.; Beltran, B.; Amirat, Y.; Yao, G.; Han, J.; Mangel, H. Second-order sliding mode control for DFIG-based wind turbines fault ride-through capability enhancement. ISA Trans. 2014, 53, 827-833. [CrossRef]

32. Evangelista, C.; Puleston, P.; Valenciaga, F.; Fridman, L.M. Lyapunov-designed super-twisting sliding mode control for wind energy conversion optimization. IEEE Trans. Ind. Electron. 2012, 60, 538-545. [CrossRef]

33. Susperregui, A.; Martinez, M.I.; Zubia, I.; Tapia, G. Design and tuning of fixed-switching-frequency second-order sliding-mode controller for doubly fed induction generator power control. IET Electr. Power Appl. 2012, 6, 696-706. [CrossRef]

34. Susperregui, A.; Martinez, M.I.; Tapia, G.; Vechiu, I. Second-order sliding-mode controller design and tuning for grid synchronisation and power control of a wind turbine-driven doubly fed induction generator. IET Renew. Power Gener. 2013, 7, 540-551. [CrossRef]

35. Martinez, M.I.; Susperregui, A.; Tapia, G. Second-order sliding-mode-based global control scheme for wind turbine-driven DFIGs subject to unbalanced and distorted grid voltage. IET Electr. Power Appl. 2017, 11, 1013-1022. [CrossRef]

36. Shah, A.P.; Mehta, A.J. Direct power control of DFIG using super-twisting algorithm based on second-order sliding mode control. In Proceedings of the 2016 14th International Workshop on Variable Structure Systems (VSS), Nanjing, China, 1-4 June 2016; pp. 136-141. [CrossRef]

37. Shah, A.P.; Mehta, A.J. Direct power control of grid-connected DFIG using variable gain super-twisting sliding mode controller for wind energy optimization. In Proceedings of the IECON 2017-43rd Annual Conference of the IEEE Industrial Electronics Society, Beijing, China, 29 October-1 November 2017; pp. 2448-2454. [CrossRef]

38. Zhang, Y.; Qu, C. Direct power control of a pulse width modulation rectifier using space vector modulation under unbalanced grid voltages. IEEE Trans. Power Electron. 2014, 30, 5892-5901. [CrossRef]

39. Seeber, R.; Horn, M. Stability proof for a well-established super-twisting parameter setting. Automatica 2017, 84, 241-243. [CrossRef]

(C) 2019 by the authors. Licensee MDPI, Basel, Switzerland. This article is an open access article distributed under the terms and conditions of the Creative Commons Attribution (CC BY) license (http://creativecommons.org/licenses/by/4.0/). 